\title{
Accurate, First-Principle Study of Electronic and Related Properties of the Ground State of $\mathrm{Li}_{2} \mathrm{Se}$
}

\author{
Abdoulaye Goita, Feng Gao, Ifeanyi H. Nwigboji, Yuriy Malozovsky, Lashounda Franklin, \\ Diola Bagayoko*
}

Department of Physics, Southern University and A\&M College, Baton Rouge, LA, USA

Email: ^Diola_Bagayoko@subr.edu

How to cite this paper: Goita, A., Gao, F., Nwigboji, I.H., Malozovsky, Y., Franklin, L. and Bagayoko, D. (2019) Accurate, First-Principle Study of Electronic and Related Properties of the Ground State of $\mathrm{Li}_{2} \mathrm{Se}$. Journal of Modern Physics, 10 , 909-921.

https://doi.org/10.4236/jmp.2019.108060

Received: May 13, 2019

Accepted: July 6, 2019

Published: July 9, 2019

Copyright (๑) 2019 by author(s) and Scientific Research Publishing Inc. This work is licensed under the Creative Commons Attribution International License (CC BY 4.0).

http://creativecommons.org/licenses/by/4.0/

\begin{abstract}
We present results from ab-initio, self-consistent calculations of electronic and related properties for the ground state of cubic lithium selenide $\left(\mathrm{Li}_{2} \mathrm{Se}\right)$. We employed a local density approximation (LDA) potential and performed computations following the Bagayoko, Zhao, and Williams (BZW) method, as enhanced by Ekuma and Franklin (BZW-EF). This method verifiably leads to the ground state of materials without employing over-complete basis sets. We present the calculated electronic energies, total and partial densities of states, effective masses, and the bulk modulus. The present calculated band structures show clearly that cubic $\mathrm{Li}_{2} \mathrm{Se}$ has a direct fundamental energy band gap of 4.065 $\mathrm{eV}$ at the $\Gamma$ point for the room temperature experimental lattice constant of $6.017 \AA$. This result is different from findings of previous density functional theory (DFT) calculations that uniformly reported an indirect band gap, from $\Gamma$ to $X$, for $\mathrm{Li}_{2} \mathrm{Se}$. We predicted a direct band gap of $4.363 \mathrm{eV}$, at the computationally determined equilibrium lattice constant of $5.882 \AA$, and a bulk modulus of $35.4 \mathrm{GPa}$. For the first time known to us, we report calculated electron and hole effective masses for $\mathrm{Li}_{2} \mathrm{Se}$. The experimental confirmation of the large, direct gap we found will point to a potential importance of this material for ultraviolet technologies and applications. Due to a lack of experimental results, most of our calculated ones in this paper are predictions for $\mathrm{Li}_{2} \mathrm{Se}$.
\end{abstract}

\section{Keywords}

Density Functional Theory, BZW-EF Method, Electronic Properties, Bulk Modulus

\section{Introduction}

Alkali metal chalcogenides $\left(\mathrm{X}_{2} \mathrm{Ch}, \mathrm{X}=\mathrm{Li}, \mathrm{Na}, \mathrm{K} ; \mathrm{Ch}=\mathrm{O}, \mathrm{S}, \mathrm{Se}, \mathrm{Te}\right)$ have high 
ionic conductivity and large fundamental energy band gaps, leading to their promising applications in power sources, fuel cells, solid state gas-detectors, ultraviolet space technology devices and photocatalysis [1] [2] [3] [4]. Compared to the extensive studies on the alkali metals oxides and sulfides, the alkali metal selenides have received much less attention. Recently, several research works have focused on lithium selenide $\left(\mathrm{Li}_{2} \mathrm{Se}\right)$ for its superionic (SI) properties. The fast $\mathrm{Li}^{1+}$-ion transport of the $\mathrm{SI} \mathrm{Li}_{2} \mathrm{Se}$ solid makes it a prime candidate for solid-state electrolytes in next generation lithium battery technologies [5]. Up until now, however, experimental studies of electronic and related properties of $\mathrm{Li}_{2} \mathrm{Se}$ are very few. As far as we know, most of the research work on $\mathrm{Li}_{2} \mathrm{Se}$ has been confined to studies of its structural properties. No experimental measurements regarding the electronic and related properties of $\mathrm{Li}_{2} \mathrm{Se}$ are available. Theoretically, only the following three research groups have performed first-principle calculations of electronic band structures of $\mathrm{Li}_{2} \mathrm{Se}$. We summarized their findings in Table 1 below. Eithiraj et al. calculated the electronic structure of $\mathrm{Li}_{2} \mathrm{Se}$, using a Tight-Binding and Linear Muffin-Tin Orbital (TB-LMTO) method [6] [7] and the local density approximation (LDA) potential of von Barth and Hedin [8] [9]. Their results show that $\mathrm{Li}_{2} \mathrm{Se}$ is an indirect band gap semiconductor, with a gap of $2.748 \mathrm{eV}$, from $\Gamma$ to $X$. Alay-e-Abbas et al. calculated the band structures of $\mathrm{Li}_{2} \mathrm{Se}$ using the Full Potential Linearized Augmented Plane Wave (FP-LAPW) method, as implemented in the WIEN2K program package [10], and density functional theory (DFT) potentials [11] [12]. Specifically, these authors employed a local density approximation (LDA), the Perdew-Burke-Ernzerhof [13] generalized gradient approximation (PBE-GGA), the Wu and Cohen [14] GGA (WU-GGA), which entails fourth-order gradient expansion of exchange energy function, and the Engel and Vosko [15] GGA (EV-GGA) potentials. The band structures calculated within the LDA, PBE-GGA, WC-GGA and EV-GGA potentials exhibit $\Gamma$ to $X$ indirect band gap values of $2.78 \mathrm{eV}, 2.93 \mathrm{eV}, 2.82 \mathrm{eV}$, and $4.08 \mathrm{eV}$, respectively.

Table 1. Previously calculated band gaps of $\mathrm{Li}_{2} \mathrm{Se}$. The computational approaches and the utilized DFT potentials are respectively in Columns I and II, while the two lowest, calculated gaps are in Columns III and IV.

\begin{tabular}{cccc}
\hline \multirow{2}{*}{ Calculation Methods } & Exchange-Correlation & \multicolumn{2}{c}{ Energy Band Gap, $\mathbf{E}_{\mathrm{g}}(\mathrm{eV})$} \\
\cline { 3 - 4 } & & Direct $(\Gamma-\Gamma)$ & Indirect $(\Gamma-\mathrm{X})$ \\
\hline FP-LAPW & LDA & $3.23[11]$ & $2.78[11]$ \\
FP-LAPW & PBE-GGA & $3.45[11]$ & $2.93[11]$ \\
FP-LAPW & WC-GGA & $3.18[11]$ & $2.82[11]$ \\
FP-LAPW & EV-GGA & $4.73[11]$ & $4.08[11]$ \\
FP-LAPW & WC-GGA & - & $2.80[15]$ \\
FP-LAPW & EV-GGA & - & $4.12[15]$ \\
FP-LAPW & mBJ & - & $4.19[15]$ \\
TB-LMTO & LDA & $3.457[8]$ & $2.748[8]$ \\
\hline
\end{tabular}


Ali et al. carried out first-principle DFT calculations of electronic properties of $\mathrm{Li}_{2} \mathrm{Se}$, using the FP-LAPW method and the WC-GGA as well as the EV-GGA potentials [16]. In their work, they utilized the recently modified Becke and Johnson (mBJ) potential [17], which is a "hybrid" potential whose amount of "exact exchange" is controlled by a parameter $c$, to improve the calculated, electronic band structure. The first-principles WC-GGA, EV-GGA and mBJ calculations by Ali et al. show that $\mathrm{Li}_{2} \mathrm{Se}$ has a $\Gamma$ to $X$ indirect band gap of $2.80 \mathrm{eV}, 4.12$ $\mathrm{eV}$, and $4.19 \mathrm{eV}$, respectively [16].

Our motivation for this work partly stems from current and potential applications of $\mathrm{Li}_{2} \mathrm{Se}$ for the next generation of battery technologies. Accurate, calculated electronic and related properties are important in informing and in guiding the development of new applications. While previous DFT calculations agreed on the indirect nature of the band gap, the resulting numerical values range from $2.748 \mathrm{eV}$ to $4.19 \mathrm{eV}$. Such a wide range points to the need for further theoretical studies of electronic and related properties of lithium selenide. The current lack of experimental studies of electronic and related properties of $\mathrm{Li}_{2} \mathrm{Se}$ is an added motivation for this work. With our distinctive computational method, we have correctly described and predicted electronic and related properties of more than 30 semiconductors [18]. These past successes portend an accurate DFT description of this material, using our BZW-EF method. We describe below, in Section 2 , the general computational approach and our distinctive method. We subsequently present our findings in Section 3 . We then provide discussions and a conclusion in Sections 4 and 5, respectively.

\section{Computational Method}

In ambient conditions, $\mathrm{Li}_{2} \mathrm{Se}$ crystallizes in a stable face center cubic (FCC) antifluorite (anti-CaF ${ }_{2}$-type) structure [19] (Space group $O_{h}^{5}-F m \overline{3} m$, No. 225), with the $\mathrm{Li}$ atoms located at $\pm(0.25,0.25,0.25)$ and the Se atoms at $(0,0,0)$ Wyckoff positions. In this work, we performed first-principle full-potential DFT calculations for the electronic properties of $\mathrm{Li}_{2} \mathrm{Se}$, using the experimental lattice constant of $6.017 \AA$ from Zintl et al. [19] and our predicted, equilibrium lattice constant. We utilized a linear combination of atomic orbitals (LACO) formalism and the BZW-EF method, which has been extensively described in several of our previous publications [18] [20] [21] [22] [23]. Our first-principle LCAO package is from the Ames laboratory of the US Department of Energy, in Ames, Iowa [24] [25]. We began the calculations with self-consistent computations for the atomic wave functions for $\mathrm{Li}^{1+}$ and $\mathrm{Se}^{2-}$ atoms. The radial parts of the atomic wave functions were expanded in terms of Gaussian functions. The $s, p$ orbitals for the cation $\mathrm{Li}^{+}$were described with 16 even-tempered Gaussian functions with respective minimum and maximum exponents of 0.2400 and $0.90 \times 10^{5}$ for the atomic potential and 0.1200 and $0.90 \times 10^{5}$ for the atomic wave functions. The self-consistent calculations for $\mathrm{Li}^{+}$led to the total charge of 2.0009, which is also the valence charge. For $\mathrm{Se}^{2-}$ the $s, p$ and $d$ orbitals were described with 24 even-tempered Gaussian functions with respective minimum and maximum 
Gaussian exponents of 0.2300 and $0.220 \times 10^{6}$ for the atomic potential and 0.1350 and $0.240 \times 10^{6}$ for the atomic functions, respectively. These Gaussian exponents led to the convergence of the atomic calculations. We utilized the Ceperley and Alder local density approximation (LDA) potential. In the iterations for self-consistency, we used a mesh of $60 \mathrm{k}$-points with proper weights in the irreducible Brillouin zone. We reached convergence for a given self-consistent calculation after 90 iterations; the criterion for convergence was that then, the difference between the potentials from the last two consecutive iterations was $10^{-4}$ or less. Further, for the production of the final, self-consistent electronic band structures, we used a total of $81 k$ points in the Brillouin zone, with the same computational errors as for the self-consistent potential calculations. Based on the above points, our computational approach is the same as those of other DFT calculations. We underscore below the critically important, distinctive feature of our computational method, with multiple, self-consistent calculations with basis sets of different sizes.

Our ab initio self-consistent calculations for the solid, with the BZW-EF method, began with a small basis set containing the minimum basis set, which is the smallest one accounting for all the electrons in the system under study, i.e., $\mathrm{Li}_{2} \mathrm{Se}$. Following this Calculation I, we augmented the basis set with one orbital representing an excited state and performed Calculation II. We graphically and numerically compared the occupied energies from Calculations I and II, with the Fermi levels set to zero. After augmenting the basis set of Calculation II with one orbital, we carried out Calculation III and compared the resulting occupied energies with those from Calculation II. In both of the preceding comparisons of occupied energies, at least some of the ones obtained with the larger basis set were lower than corresponding ones from the immediately preceding calculation (with a smaller basis set). We continued this process of augmenting the basis set and of performing self-consistent calculations until three consecutive ones led to the same occupied energies. The perfect superposition of these occupied energies is the criterion or proof that these calculations produced the absolute minima of the occupied energies, i.e., the ground state of the system.

Let $\mathrm{N}$ be the number of the first of these three calculations to reach the ground state. We dubbed the basis set of this calculation as the optimal basis set, i.e., the smallest basis set that leads to the ground state upon the attainment of self-consistency. Calculations $(\mathrm{N}+1),(\mathrm{N}+2)$ and other with larger, augmented basis sets produced (a) the same charge density, (b) the same Hamiltonian, and (c) the same occupied energies as respectively obtained with Calculation $\mathrm{N}$. We distinguish the Hamiltonian from the Hamiltonian matrix that changes with the size of the basis set. Despite (a) through (c) above, some unoccupied energies from calculations $(\mathrm{N}+1),(\mathrm{N}+2)$ and others with larger, augmented basis sets, were generally lower than corresponding ones obtained in Calculation N. Given that the Hamiltonian did not change from that of Calculation N, any eigenvalues that deviate from (i.e., are lower than) their corresponding values resulting from Calculation $\mathrm{N}$ are clearly unphysical. Another proof of this assertion stems from 
the second corollary ${ }^{18}$ of the first DFT theorem: According to it, the spectrum of the ground state Hamiltonian is a unique functional of the ground state charge density. Hence, if an eigenvalue from Calculation $(\mathrm{N}+1)$ or higher is different (lower than) its corresponding value obtained in Calculation $\mathrm{N}$, then the new value no longer belongs to the spectrum of the Hamiltonian-as the charge density did not change. In summary, Calculation $\mathrm{N}$ is the only one providing the true DFT description of the material; the resulting eigenvalues possess the full physical content of the DFT, unlike eigenvalues resulting from self-consistent iterations with a single basis set. These iterations produce stationary solutions among an infinite number of such solutions. Our generalized minimization of the energy functional of the Hamiltonian, using successive, self-consistent calculations, verifiably reaches the true ground state of the system-instead of an arbitrary, stationary solution unwittingly confused with the ground state.

\section{Results}

Table 2 shows the successive calculations inherent to the implementation of the BZW-EF method. Calculation III-V were the first ones to reach the ground state of the system, as explained above in the method section. As per the explanations in this method section, Calculation III is the one providing the DFT description of $\mathrm{Li}_{2} \mathrm{Se}$. Table 2 also shows the specific orbitals for the two ionic species, the total number of valence functions (with the number of orbitals for $\mathrm{Li}^{+}$counted twice for $\mathrm{Li}_{2} \mathrm{Se}$ ), and the calculated band gaps at the $\Gamma$ point, from $\Gamma$ to $\mathrm{X}$, and from $\Gamma$ to $\mathrm{K}$. The superscript of zero for an orbital signifies that it represents an unoccupied state, i.e., an excited state.

Figure 1 shows the calculated, electronic band structures of $\mathrm{Li}_{2} \mathrm{Se}$ along high symmetry $\mathrm{k}$ points in the irreducible Brillouin zone, as obtained in Calculations III and IV of the BZW-EF method.

Table 2. Successive, self-consistent calculations in the implementation of the BZW-EF method of the generalized minimization of the energy. The band gaps are in the last three columns, in $\mathrm{eV}$. For the total number of valence functions, the number of orbitals on $\mathrm{Li}^{1+}$ is counted twice. The smallest band gap is direct, for Calculations I-V, it is indirect for Calculation VI. Calculation III provides the DFT description of $\mathrm{Li}_{2} \mathrm{Se}$; it is the first of three consecutive ones leading to the same absolute minima of the occupied energies (i.e., the ground state, with a direct band gap of $4.065 \mathrm{eV}$ at $\Gamma$ ).

\begin{tabular}{ccccccc}
\hline Cal. No. & $\begin{array}{c}\text { Orbitals } \\
\text { for } \mathrm{Li}^{1+}\end{array}$ & $\begin{array}{c}\text { Orbitals for valence } \\
\text { state of } \mathrm{e}^{2-}\end{array}$ & $\begin{array}{c}\text { No. of } \\
\text { Valence } \\
\text { Functions }\end{array}$ & $\begin{array}{c}\text { Gap } \\
(\text { at } \Gamma) \text { in } \mathrm{eV}\end{array}$ & $\begin{array}{c}\text { Gap }(\Gamma-\mathrm{X}) \\
\text { in } \mathrm{eV}\end{array}$ & $\begin{array}{c}\text { Gap } \\
(\Gamma-\mathrm{K}) \text { in } \mathrm{eV}\end{array}$ \\
\hline I & $1 \mathrm{~s}^{2} 2 \mathrm{~s}^{0} 2 \mathrm{p}^{0}$ & $3 \mathrm{~s}^{2} 3 \mathrm{p}^{6} 3 \mathrm{~d}^{10} 4 \mathrm{~s}^{2} 4 \mathrm{p}^{6}$ & 46 & 4.111 & 4.551 & 5.427 \\
II & $1 \mathrm{~s}^{2} 2 \mathrm{~s}^{0} 2 \mathrm{p}^{0}$ & $3 \mathrm{~s}^{2} 3 \mathrm{p}^{6} 3 \mathrm{~d}^{10} 4 \mathrm{~s}^{2} 4 \mathrm{p}^{6} 4 \mathrm{~d}^{0}$ & 56 & 4.096 & 4.536 & 5.412 \\
III & $1 \mathrm{~s}^{2} 2 \mathrm{~s}^{0} 2 \mathrm{p}^{0} 3 \mathrm{p}^{0}$ & $3 \mathrm{~s}^{2} 3 \mathrm{p}^{6} 3 \mathrm{~d}^{10} 4 \mathrm{~s}^{2} 4 \mathrm{p}^{6} 4 \mathrm{~d}^{0}$ & 68 & 4.065 & 4.395 & 5.195 \\
IV & $1 \mathrm{~s}^{2} 2 \mathrm{~s}^{0} 2 \mathrm{p}^{0} 3 \mathrm{p}^{0} 3 \mathrm{~s}^{0}$ & $3 \mathrm{~s}^{2} 3 \mathrm{p}^{6} 3 \mathrm{~d}^{10} 4 \mathrm{~s}^{2} 4 \mathrm{p}^{6} 4 \mathrm{~d}^{0}$ & 72 & 4.023 & 4.394 & 5.172 \\
V & $1 \mathrm{~s}^{2} 2 \mathrm{~s}^{0} 2 \mathrm{p}^{0} 3 \mathrm{p}^{0} 3 \mathrm{~s}^{0}$ & $3 \mathrm{~s}^{2} 3 \mathrm{p}^{6} 3 \mathrm{~d}^{10} 4 \mathrm{~s}^{2} 4 \mathrm{p}^{6} 4 \mathrm{~d}^{0} 5 \mathrm{p}^{0}$ & 78 & 3.940 & 4.221 & 4.967 \\
VI & $1 \mathrm{~s}^{2} 2 \mathrm{~s}^{0} 2 \mathrm{p}^{0} 3 \mathrm{p}^{0} 3 \mathrm{~s}^{0} 3 \mathrm{~s}^{2} 3 \mathrm{p}^{6} 3 \mathrm{~d}^{10} 4 \mathrm{~s}^{2} 4 \mathrm{p}^{6} 4 \mathrm{~d}^{0} 5 \mathrm{p}^{0} 5 \mathrm{~s}^{0}$ & 80 & 3.923 & 3.900 & 4.883 \\
\hline
\end{tabular}




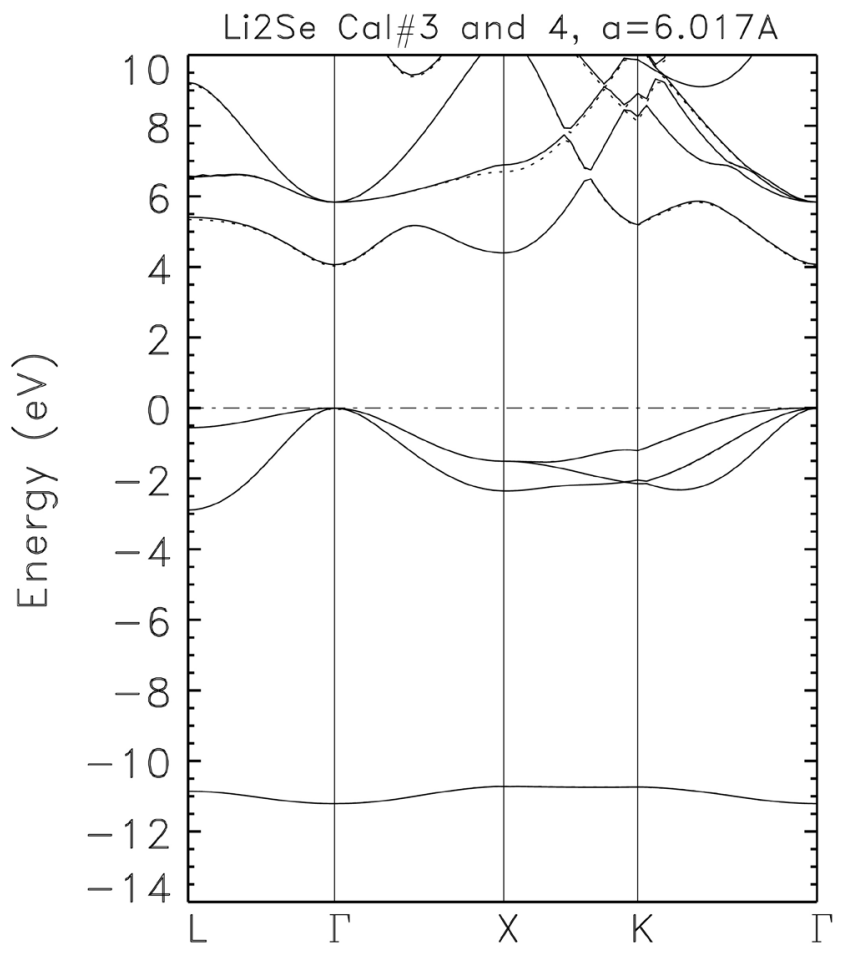

Figure 1. Calculated band structure of $\mathrm{Li}_{2} \mathrm{Se}$, as obtained in Calculations III and IV of the BZW-EF method. The occupied energies from the two calculations are perfectly superimposed. No calculations with basis sets resulting from augmenting that of Calculation III produce occupied energies lower than their corresponding values from Calculation III.

From this figure and the content of Table 2 above, we conclude that $\mathrm{Li}_{2} \mathrm{Se}$ is a direct band gap semiconductor; this result is in stark disagreement with the previously reported DFT band gaps, in Table 1, that are uniformly given that all of these previous gaps are indirect. The top of the valence band and the bottom of the conduction bands are both at the $\Gamma$ point. From the results of Calculation III, performed with the optimal basis sets, the calculated DFT band gaps of the material are $4.065 \mathrm{eV}($ at $\Gamma), 4.395(\Gamma-\mathrm{X})$ and $5.195 \mathrm{eV}(\Gamma-\mathrm{K})$, respectively.

In Table 3, we list illustrative, calculated, electronic energies for $\mathrm{Li}_{2} \mathrm{Se}$ at high symmetry points $(\Gamma, X, K$, and $L)$ in the Brillouin zone. These energies are expected to be useful in comparisons of our findings with future, experimental results. Such results include direct, optical transition energies and various X-ray and ultraviolet (UV) spectroscopic measurements.

Figure 2 shows the total electronic density of states (DOS) derived from the bands produced in Calculation 3, with the optimal basis set. The inset presents the magnified DOS in the vicinity of the band gap. This inset suggests a relatively sharp absorption edge starting around $4 \mathrm{eV}$. The total valence bandwidth is about $11.22 \mathrm{eV}$, from the DOS figure, and $11.21 \mathrm{eV}$, from the above table of eigenvalues. From the DOS figure, the width of the lowest laying valence band is $0.456 \mathrm{eV}$, while that of the group of upper valence bands is about $2.93 \mathrm{eV}$. The peaks in the DOS for the conduction band are at $5.74 \mathrm{eV}, 6.653 \mathrm{eV}, 8.86 \mathrm{eV}$, and $9.47 \mathrm{eV}$, according to Figure 2. 
Table 3. At the room temperature lattice constant of $6.017 \AA$, the calculated eigenvalues (in $\mathrm{eV}$ ), along high symmetry points, for rock salt $\mathrm{Li}_{2} \mathrm{Se}$ are shown below. They resulted from Calculation III, the first to reach the ground state of the system.

\begin{tabular}{cccc}
\hline$\Gamma$-point & X-point & K-point & L-point \\
\hline 14.367 & 15.494 & 14.907 & 13.690 \\
12.251 & 12.993 & 13.748 & 13.109 \\
12.251 & 12.993 & 10.588 & 11.760 \\
12.251 & 11.615 & 10.353 & 11.760 \\
5.836 & 10.509 & 9.875 & 9.221 \\
5.836 & 10.509 & 8.918 & 6.573 \\
5.836 & 6.889 & 8.264 & 6.548 \\
4.065 & 4.395 & 5.194 & 14.907 \\
0.000 & -1.509 & -1.207 & -0.557 \\
-0.003 & -1.509 & -2.041 & -0.557 \\
-0.003 & -2.347 & -2.142 & -2.889 \\
-11.211 & -10.724 & -10.739 & -10.857 \\
\hline
\end{tabular}

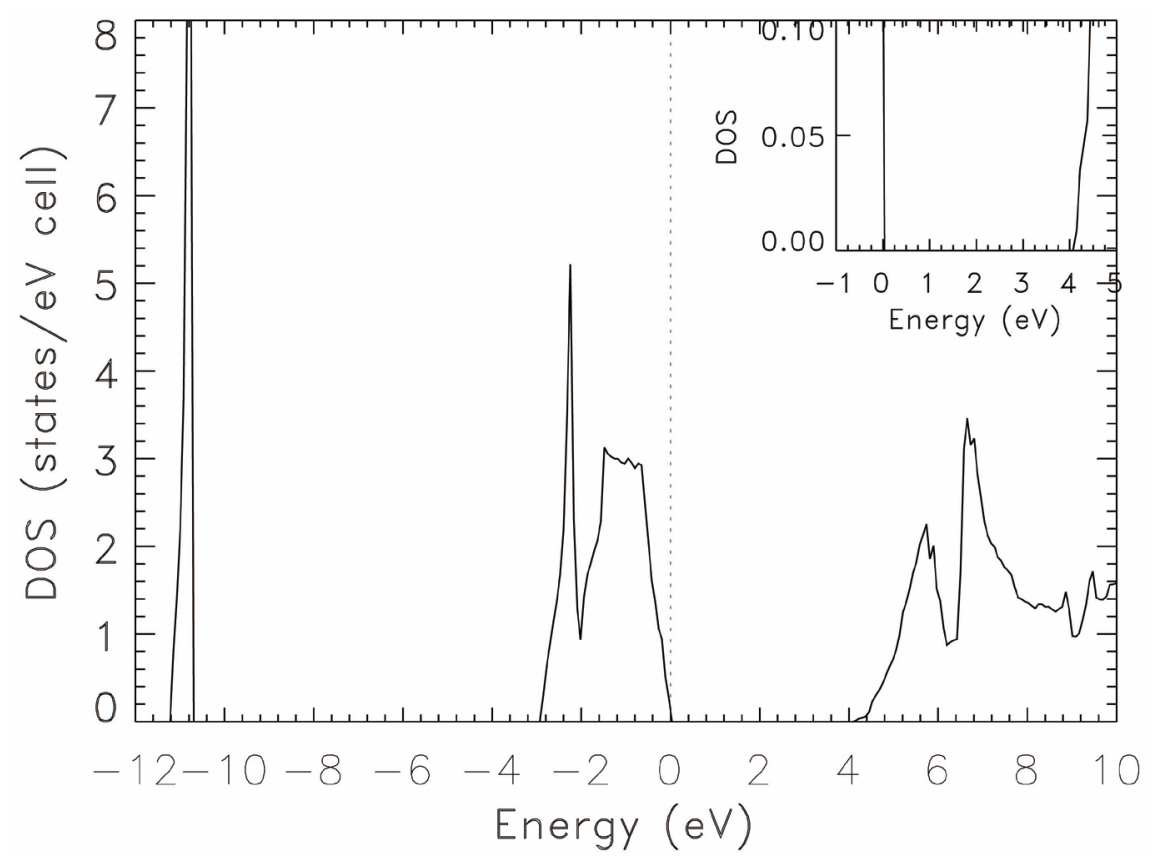

Figure 2. Calculated, total density of states (DOS) for $\mathrm{Li}_{2} \mathrm{Se}$, as derived from the bands resulting from Calculation III, the first to reach the ground state. The vertical line at zero indicates the position of the Fermi level. The inset suggests a relatively sharp absorption edge in the vicinity of $4 \mathrm{eV}$.

We present the electronic, partial DOS (pDOS) in Figure 3. These densities are also derived from the bands obtained with the optimal basis set. From the pDOS, we see that the valence band of $\mathrm{Li}_{2} \mathrm{Se}$ is almost exclusively composed of Se $\mathrm{s}$ and $\mathrm{p}$ states. In other words, these bands are described by the $\mathrm{s}$ and $\mathrm{p}$ atomic 


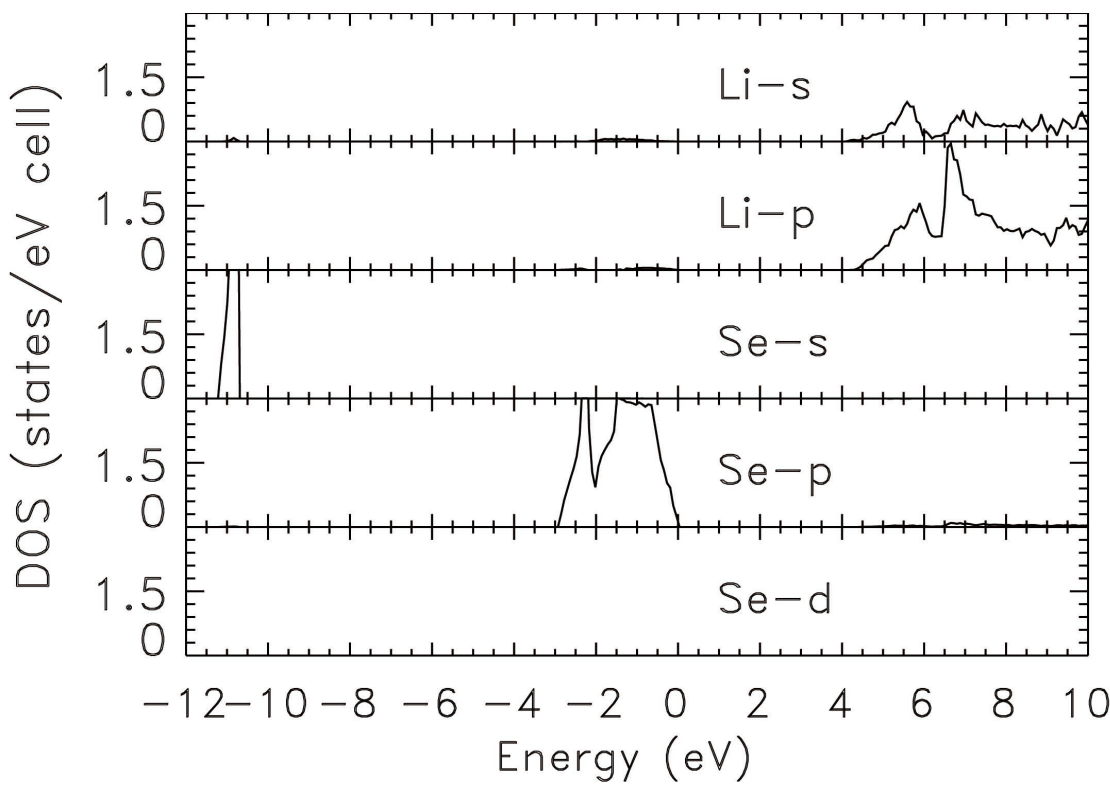

Figure 3. Calculated, partial densities of states (pDOS) for $\mathrm{Li}_{2} \mathrm{Se}$, as derived from the band resulting from Calculation, the first one to reach the ground state of the system. Zero on the horizontal axis indicates the position of the Fermi level.

orbitals on Se. More specifically, the lowest laying valence band is dominated by Se s state, with a feint contribution from Li-s. The uppermost group of valence bands is from Se $\mathrm{p}$ state. The conduction bands are mainly from the hybridization of Li p and s, with the domination of the former. Se d (3d), even though treated as valence states in the calculations, turned out to belong to the core states. The pDOS figure shows that $\mathrm{Se} 4 \mathrm{~d}$ contributes to much higher, excited states. The above features of the band structure can be measured in various $\mathrm{X}$-ray and UV spectroscopic investigations, as was the case for our calculations of electronic and related properties of wurtzite aluminum nitride [26].

The electrical conductivities, and transport and other related properties of materials require an accurate and detailed knowledge of effective masses. As per the content of Table 4, we have performed calculations of electron effective masses around the minimum of the conduction band, at the $\Gamma$ point, and around the next, lowest conduction band minimum at the X-point. We have calculated the effective masses of the light and the two heavy holes at the top of the valence band, at the $\Gamma$-point. We list these calculated, effective masses in Table 4 below, for various directions, in units of the mass of the electron $\left(\mathrm{m}_{\mathrm{o}}\right)$. The effective masses of heavy Hole 1 and heavy Hole 2 are equal, except in the $(\Gamma-\mathrm{K})_{110}$ direction. Their difference in that direction is due to the splitting of the bands in the $(\Gamma-\mathrm{K})_{110}$ direction by the Coulomb crystal field. The hole effective masses are much more anisotropic than those for the electron. The calculated electron effective mass for antifluorite $\mathrm{Li}_{2} \mathrm{Se}$, in the vicinity of the $\Gamma$ point, is nearly isotropic and is equal to $0.352 \mathrm{~m}_{\mathrm{o}}$. The electron effective masses at the $\mathrm{X}$ point are essentially anisotropic, with the longitudinal electron effective mass of $0.647 \mathrm{~m}_{\mathrm{o}}$ in the $\mathrm{X}$ to $\Gamma$ direction, with the transverse electron effective mass of $0.413 \mathrm{~m}_{\mathrm{o}}$ in 
the $\mathrm{X}$ to $\mathrm{K}$ direction, and of $0.464 \mathrm{~m}_{\mathrm{o}}$ in the $\mathrm{X}$ to $\mathrm{U}$ direction. The value of the heavy Hole 1 effective mass is $2.33 \mathrm{~m}_{\mathrm{o}}$ in the $\Gamma$ to $\mathrm{L}$ direction $(\Gamma-\mathrm{L})_{111}, 1.17 \mathrm{~m}_{\mathrm{o}}$ in the $\Gamma$ to $\mathrm{X}$ direction $(\Gamma-\mathrm{X})_{100}$, and $1.53 \mathrm{~m}_{\mathrm{o}}$ in the $\Gamma$ to $\mathrm{K}$ direction $(\Gamma-\mathrm{K})_{110}$. This hole effective mass is strongly anisotropic. The heavy Hole 2 effective mass in the $(\Gamma-\mathrm{K})_{110}$ direction is $0.989 \mathrm{~m}_{\mathrm{o}}$. The effective masses of light Hole are $0.292 \mathrm{~m}_{\mathrm{o}}$ in the $(\Gamma-\mathrm{L})_{111}$ direction, $0.515 \mathrm{~m}_{\mathrm{o}}$ in the $(\Gamma-\mathrm{X})_{100}$ direction, and $0.343 \mathrm{~m}_{\mathrm{o}}$ in the $(\Gamma-\mathrm{K})_{110}$ direction. We found no experimental values for these effective masses. We expect future measurements to confirm our predictions in Table 4.

The graph of the total energy versus the lattice constant is shown in Figure 4

Table 4. Calculated, effective masses for antifluorite $\mathrm{Li}_{2} \mathrm{Se}$, in units of free electron mass $\left(m_{\mathrm{o}}\right) . \mathrm{M}_{\mathrm{e}}$ indicates an electron effective mass at $\Gamma$ or at X.; $\mathrm{M}_{\mathrm{hh}}$ and $\mathrm{M}_{\mathrm{lh}}$ denote the heavy and light hole effective masses, respectively.

\begin{tabular}{cc}
\hline Types and Directions of Effective Masses & Values of Effective Masses $\left(\mathrm{m}_{\mathrm{o}}\right)$ \\
\hline $\mathrm{M}_{\mathrm{e}}$ & 0.352 \\
$\mathrm{M}_{\mathrm{e}}(\mathrm{X}-\Gamma)$ Longitudinal & 0.647 \\
$\mathrm{M}_{\mathrm{e}}(\mathrm{X}-\mathrm{K})$ Transverse & 0.413 \\
$\mathrm{M}_{\mathrm{e}}(\mathrm{X}-\mathrm{U})$ Transverse & 0.464 \\
$\mathrm{M}_{\mathrm{hh} 1}(\Gamma-\mathrm{L})_{111}$ & 2.33 \\
$\mathrm{M}_{\mathrm{hh} 1}(\Gamma-\mathrm{X})_{100}$ & 1.17 \\
$\mathrm{M}_{\mathrm{hh} 1}(\Gamma-\mathrm{K})_{110}$ & 1.53 \\
$\mathrm{M}_{\mathrm{hh} 2}(\Gamma-\mathrm{K})_{110}$ & 0.989 \\
$\mathrm{M}_{\mathrm{lh}}(\Gamma-\mathrm{L})_{111}$ & 0.292 \\
$\mathrm{M}_{\mathrm{lh}}(\Gamma-\mathrm{X})_{100}$ & 0.515 \\
$\mathrm{M}_{\mathrm{lh}}(\Gamma-\mathrm{K})_{110}$ & 0.343 \\
\hline
\end{tabular}

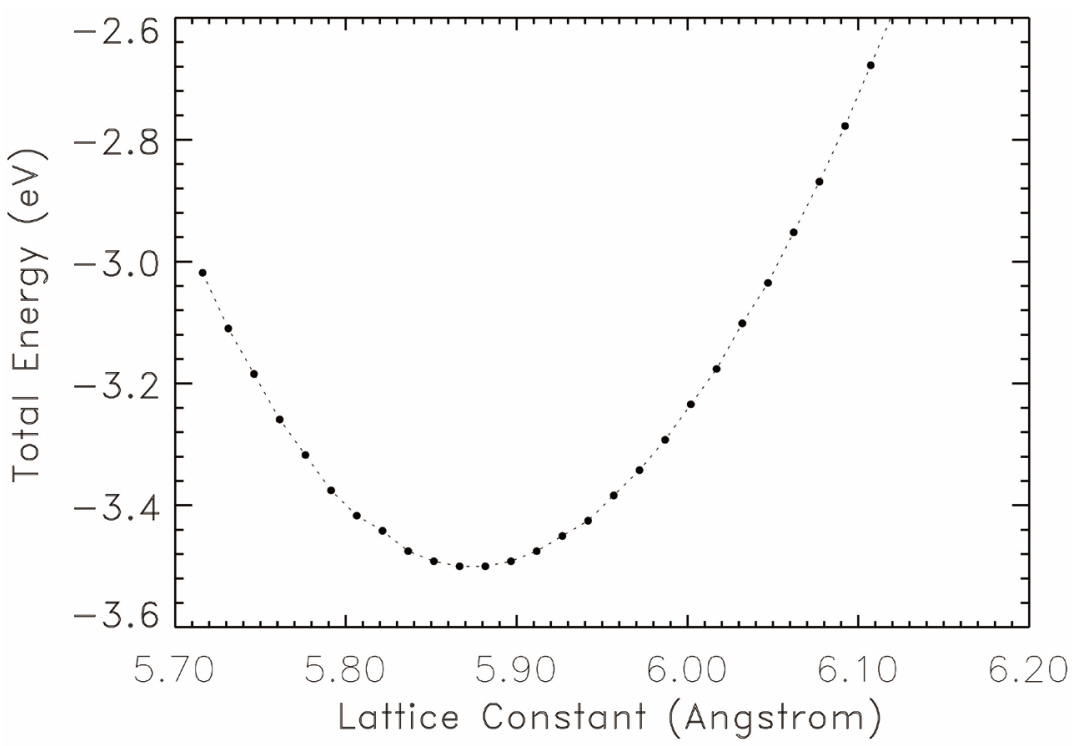

Figure 4. The total energy per unit cell, for $\mathrm{Li}_{2} \mathrm{Se}$. The minimum of the curve occurs at a $=5.882 \AA$. The predicted, direct, band gap $(\Gamma-\Gamma)$ at this lattice constant is $4.363 \mathrm{eV}$. 
below. The range of the lattice constant in which our total energy values were obtained is 5.70 to $6.20 \AA$. The minimum of the total energy curve is at $5.882 \AA$, which is our predicted equilibrium lattice constant. With this lattice constant, we predicted at zero temperature band gap of $4.363 \mathrm{eV}$ at the $\Gamma$ point, larger than the room temperature value by $0.298 \mathrm{eV}$. Our calculated bulk modulus is 35.4 $\mathrm{GPa}$; it is the same as the finding of Ali et al. [16] and is slightly larger than the calculated result of $34.72 \mathrm{GPa}$ of Alay-e-Abbas [11] [12]. No experimental measurements for the bulk modulus of $\mathrm{Li}_{2} \mathrm{Se}$ are available for comparison.

\section{Discussion}

The following discussion is guided by the fact that our calculations, as explained in the section on our method, 1) verifiably attained the ground state of the system 2) while avoiding over-complete basis sets. The latter feature guarantees that spuriously low, unoccupied energies are not in the spectrum of the ground state Hamiltonian. The two features further guarantee that the eigenvalues obtained with the optimal basis set, defined earlier, possess the full, physical content of DFT. The claim in the literature that DFT eigenvalues do not have any particular physical meaning does not apply to our findings that are true ground state results.

As shown in Table 1, all previous DFT calculations produced indirect band gaps for $\mathrm{Li}_{2} \mathrm{Se}$, in stark contrast to our calculated, direct $(\Gamma-\Gamma)$ band gaps of 4.065 $\mathrm{eV}$ and $4.363 \mathrm{eV}$, respectively, obtained with room temperature and the equilibrium lattice constants. Additionally, while three of the previous GGA results $(4.08-4.19 \mathrm{eV})$ are numerically close to ours, the latter is larger by 1.135 or more than five (5) GGA and LDA results in Table 1. The excellent agreement between our previous results and corresponding, experimental ones [8] portends a future, experimental confirmation of our findings. Such a confirmation will point to extensive, potential importance of $\mathrm{Li}_{2} \mathrm{Se}$ in various ultraviolet technologies and applications.

Most DFT calculations in the literature perform self-consistent iterations with a single basis set to produce results that are assumed to describe the ground state of the system. Such a single basis is deliberately chosen to be large, more often than not. This choice is to avoid possibilities for the basis set to be incomplete, i.e., not large enough in size (number of functions) or not rich enough in radial and angular features to accommodate the redistribution of the charge density in the formation of the system under study. As explained in our method section, large basis sets that contain the optimal one can lower some unoccupied energies; the larger the basis set, the larger the lowering is.

Hence, we should expect single basis set calculations by different authors to produce different underestimates of the band gap of a semiconductor, even if they employ the same DFT potential and similar computational approaches. The outcomes of our Calculation VI illustrate the point. Let us first recall that calculations with large basis sets resulting from an augmentation of the optimal basis set do not lower any occupied energies from their value obtained with the op- 
timal basis set. Additionally, if such basis sets are not significantly larger than the optimal one, they also reproduce low laying, unoccupied energies obtained with the optimal basis set. The content of Figure 1 shows that the low laying, unoccupied energies produced by Calculation IV are the same as those from Calculation III, up to $+6 \mathrm{eV}$. The values of the band gaps resulting from Calculation VI, whose basis set contains 12 more functions than the optimal one, illustrate the point. This calculation not only reduced the band gaps from their values obtained in Calculation III, but also it resulted in an indirect $(\Gamma-\mathrm{X})$ band gap. The latter feature is in qualitative agreement with the findings of the previous DFT calculations shown in Table 1; we presume that these single basis set calculations most likely utilized relatively large basis sets.

\section{Conclusion}

In summary, we performed first principle, self-consistent calculations of electronic, transport, and bulk properties of cubic antifluorite lithium selenide $\left(\mathrm{Li}_{2} \mathrm{Se}\right)$, using a local density approximation (LDA) potential. As per the BZW-EF method, our implementation of the linear combination of atomic orbitals entailed the performance of successive, self-consistent calculations with increasingly large basis sets. We obtain the basis set of a calculation, except for the first one that has a small basis set, by augmenting the basis set of the immediately preceding calculation with one orbital. This generalized minimization of the energy not only reached the ground state, but also does so without employing over-complete basis sets that tend to lower, unphysically, some unoccupied energies. This fact suggests that the widespread underestimation of the band gaps of semiconductors and insulators, by DFT calculations, may be due to this spurious lowering of unoccupied energies. Our calculated, indirect band gap of $\mathrm{Li}_{2} \mathrm{Se}$, at room temperature, is $4.065 \mathrm{eV}$. This result is in stark contrast with those from previous DFT calculations that found an indirect band gap. The accurate results we obtained for more than 30 semiconductors are the basis for us to expect a future, experimental confirmation of our results for the energy bands, the densities of states, effective masses, and the bulk modulus of $\mathrm{Li}_{2} \mathrm{Se}$.

\section{Acknowledgements}

This research work was funded in part by the US Department of Energy (DOE), National Nuclear Security Administration (NNSA) (Award No. DE-NA0003679), the National Science Foundation (NSF, Award no, 1503226DE), LaSPACE, and LONI-SUBR.

\section{Conflicts of Interest}

The authors declare no conflicts of interest regarding the publication of this paper.

\section{References}

[1] Minami, T., Hayashi, A. and Tatsumisago, M. (2000) Solid State Ionics, 136-137, 
1015-1023. https://doi.org/10.1016/S0167-2738(00)00555-5

[2] Bisero, D., Oerle, B.M.V., Ernst, G.J., Verschuur, J.W.J. and Witteman, W.J. (1996) Applied Physics Letters, 69, 3641. https://doi.org/10.1063/1.117009

[3] Bisero, D., Oerle, B.M.V., Ernst, G.J., Verschuur, J.W.J. and Witteman, W.J. (1997) Journal of Applied Physics, 82, 1384. https://doi.org/10.1063/1.365915

[4] Hull, S., Farley, T.W.D., Hayes, W. and Hutchings, M.T. (1988) Journal of Nuclear Materials, 160, 125. https://doi.org/10.1016/0022-3115(88)90039-6

[5] Dumett Torres, D. and Jain, P.K. (2018) The Journal of Physical Chemistry Letters, 9, 1200. https://doi.org/10.1021/acs.jpclett.8b00236

[6] Andersen, O.K. (1975) Physical Review B, 12, 3060. https://doi.org/10.1103/PhysRevB.12.3060

[7] Andersen, O.K. and Jepsen, O. (1984) Physical Review Letters, 53, 2571. https://doi.org/10.1103/PhysRevLett.53.2571

[8] Eithiraj, R.D., Jaiganesh, G. and Kalpana, G. (2009) International Journal of Modern Physics B, 23, 5027-5037. https://doi.org/10.1142/S0217979209052418

[9] Barth, U.V. and Hedin, L. (1972) Journal of Physics C: Solid State Physics, 5, 1629-1642. https://doi.org/10.1088/0022-3719/5/13/012

[10] Blaha, P., Schwarz, K., Madsen, G.K.H., Kvasnicka, D. and Luitz, J. (2001) WIEN2K: An Augmented Plane Wave + Local Orbitals Program for Calculating Crystal Properties. Karlheinz Schwarz, Techn. Universität Wien, Wien.

[11] Alay-e-Abbas, S.M., Sabir, N., Saeed, Y. and Shaukat, A. (2010) Journal of Alloys and Compounds, 503, 10-18. https://doi.org/10.1016/j.jallcom.2010.05.014

[12] Alay-e-Abbas, S.M., Sabir, N., Saeed, Y. and Shaukat, A. (2011) International Journal of Modern Physics B, 25, 3911-3925. https://doi.org/10.1142/S021797921110093X

[13] Perdew, J.P., Burke, K. and Ernzerhof, M. (1996) Physical Review Letters, 77, 3865-3868. https://doi.org/10.1103/PhysRevLett.77.3865

[14] Wu, Z. and Cohen, R.E. (2006) Physical Review B, 73, Article ID: 235116.

[15] Engel, E. and Vosko, S.H. (1993) Physical Review B, 47, Article ID: 13164. https://doi.org/10.1103/PhysRevB.47.13164

[16] Ali, R., Khanata, R., Amin, B., Murtaza, G. and Bin Omran, S. (2013) International Journal of Modern Physics B, 27, Article ID: 1350170. https://doi.org/10.1142/S0217979213501701

[17] Becke, A.D. and Johnson, E.R. (2006) The Journal of Chemical Physics, 124, Article ID: 221101. https://doi.org/10.1063/1.2213970

[18] Bagayoko, D. (2014) AIP Advances, 4, Article ID: 127104. https://doi.org/10.1063/1.4903408

[19] Zintl, E., Harder, A. and Dauth, B. (1934) Zeitschrift für Elektrochemie und angewandte physikalische Chemie, 40, 588-593.

[20] Bagayoko, D., Zhao, G.L., Fan, J.D. and Wang, J.T. (1998) Journal of Physics. Condensed Matter, 10, 5645-5655. https://doi.org/10.1088/0953-8984/10/25/014

[21] Zhao, G.L., Bagayoko, D. and Williams, T.D. (1999) Physical Review B, 60, 1563. https://doi.org/10.1103/PhysRevB.60.1563

[22] Franklin, L., Ekuma, C.E., Zhao, G.L. and Bagayoko, D. (2013) Journal of Physics and Chemistry of Solids, 74, 729-736. https://doi.org/10.1016/j.jpcs.2013.01.013

[23] Chinedu, E.E. and Diola, B. (2011) Japanese Journal of Applied Physics, 50, Article 
ID: 101103 .

[24] Feibelman, P.J., Appelbaum, J.A. and Hamann, D.R. (1979) Physical Review B, 20, 1433-1443. https://doi.org/10.1103/PhysRevB.20.1433

[25] Harmon, B.N., Weber, W. and Hamann, D.R. (1982) Physical Review B, 25, 1109. https://doi.org/10.1103/PhysRevB.25.1109

[26] Nwigboji, I.H., Ejembi, J.I., Malozovsky, Y., Khamala, B., Franklin, L., Zhao, G., Ekuma, C.E. and Bagayoko, D. (2015) Materials Chemistry and Physics, 157, 80-86. https://doi.org/10.1016/j.matchemphys.2015.03.019 